$\mathrm{M}$ ost of the planets in our Solar System, Earth among them, have magnetic fields that originate from self-sustaining dynamos; so, too, do the jovian satellites Io and Ganymede, according to the results from the Galileo mission ${ }^{1}$. Planetary magnetism is a common phenomenon because only three basic ingredients are needed for a dynamo: a sufficiently large volume of electrically conducting fluid somewhere in the planet's interior, an energy source such as convection to circulate the fluid, and planetary rotation to organize the resulting fluid motions. That sounds simple enough. But the inner workings of planetary dynamos have remained stubbornly opaque, mainly because the requirement of a large volume of conducting fluid has prevented the construction of laboratory-scale dynamos and, until recently, has permitted only rudimentary numerical simulations.

On page 371 of this issue ${ }^{2}$, Kuang and Bloxham report on numerical models of convection-driven dynamos that successfully reproduce many of the observed characteristics of the Earth's magnetic field. Perhaps the most fundamental result is that their model generates an external field dominated by an axial dipole component, like that of the Earth, with an intensity close to the present-day geomagnetic dipole moment. The Kuang-Bloxham dynamo also exhibits westward drift of the non-dipole magnetic field, much like the historically documented westward drift of the geomagnetic field.

Publication of this geodynamo simulation follows on the heels of another successful numerical model by Glatzmaier and Roberts ${ }^{3}$. The two dynamos operate under broadly similar physical conditions in terms of convective energy, rotation rate and fluid properties, and they produce comparable external magnetic fields. It is only when they are sliced open to reveal their internal dynamics that important differences appear. It turns out that these differences hold the key to understanding the dynamics of the Earth's core. Fortunately, predictions can be made from the Kuang-Bloxham and the Glatzmaier-Roberts dynamos that allow them to be tested against each other.

Both of them indicate that the solid iron inner core, despite its relatively small size, plays a vital role in the dynamics of the core as a whole. First, it is an important energy source; crystallization of iron at the inner-outer core boundary releases both latent heat and light constituents, driving convection in the liquid outer core. Second, the inner core presents a mechanical barrier to flow in the liquid outer core. It is how the liquid responds to this barrier that leads to major differences between the dynamo models.

Barriers to flow in the atmosphere and ocean are common because of stable density

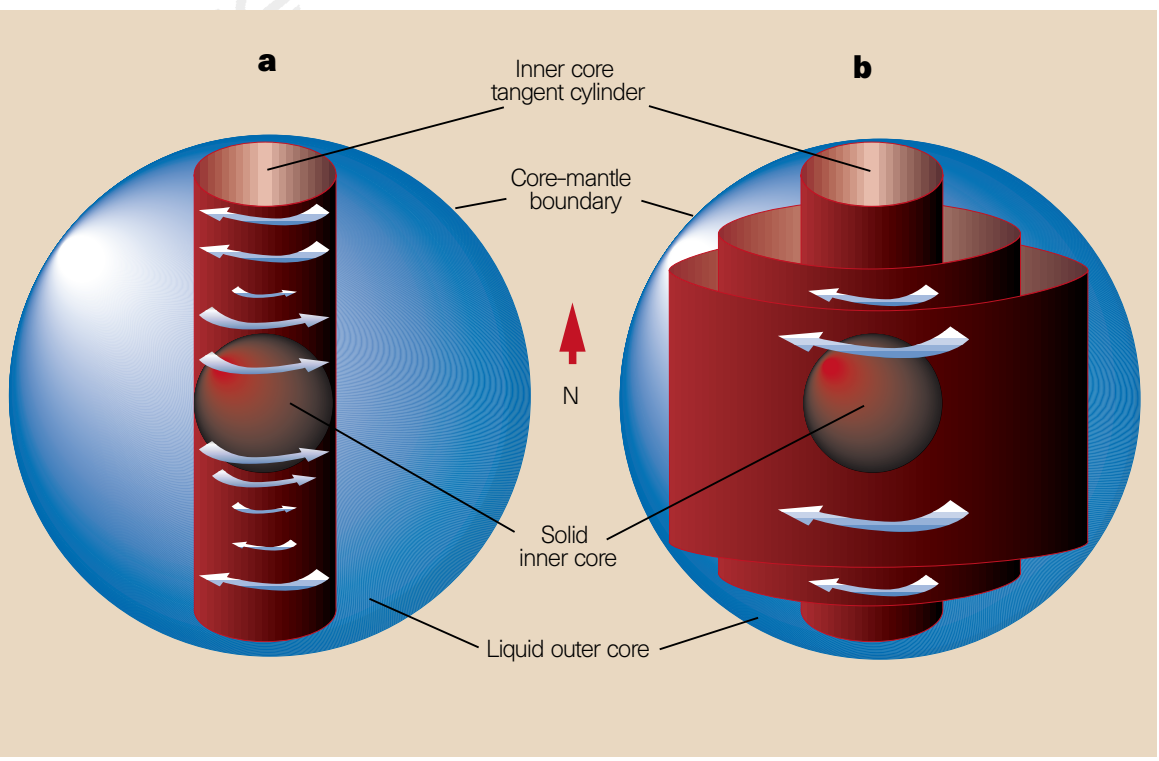

Figure 1 Alternative models of the primary azimuthal flow in the Earth's liquid outer core, which stem from setting different boundary conditions for the interaction between the inner and outer cores. a, Polar vortex flow inside the inner-core tangent cylinder, as surmised by Glatzmaier and Roberts ${ }^{3}$. b, Differential rotation outside the tangent cylinder, the result obtained by Kuang and Bloxham $^{2}$. Dynamo models with polar vortices account for the observed super-rotation of the solid inner core, whereas models with differential rotation outside the tangent cylinder account for the geomagnetic westward drift.

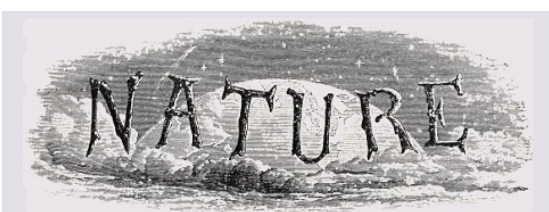

100 YEARS AGO

Professor Newcomb therefore concluded: "Either the bodies which compose our universe are vastly more massive and numerous than telescopic examination seems to indicate, or 1830 Groombridge is a runaway star, flying on a boundless course through infinite space with such momentum that the attraction of all the bodies of the universe can never stop it." A new contribution to this inquiry was read recently before the American Philosophical Society by Mr. Luigi D'Auria .... In this paper it is shown that, "given the ether the density as estimated by Maxwell, and the power of attracting matter by gravity, a body placed within the sphere of ether containing all the stars of the visible universe, and at a distance from the centre of such sphere equal to that passed over by light in 2200 years, would pass this centre with a velocity equal to that of the star 1830 Groombridge, taking into account the attraction of the ether alone; and such body would oscillate about the same centre, rectilinearly, with a period of a little over seven million years, which would be also the period of oscillation of every other star." Mr d'Auria recognises that some other, and unquestionable, cause may eventually prove to be responsible for stellar proper motions, nevertheless he thinks his results are worth putting on record.

From Nature 23 September 1897.

50 YEARS AGO

In 1681 Papin invented his "New Digester or Engine for Softening Bones", in which animal products are stewed by heat under pressure; this was a great advance in technique fraught with many valuable applications in subsequent years. To limit the pressure in the digester and so avoid bursting it, Papin employed the lever safety valve, with the invention of which he is not quite correctly credited; what is certain is that he was the first to illustrate it in print.... John Evelyn, in his "Diary" under date April 5, 1682, tells with gusto and appreciation of a supper cooked with the digester and partaken of in company with other fellows of the Royal Society after a meeting. He remarks: "This philosophical supper caus'd much mirth amongst us and exceedingly pleas'd all the company", as one can well imagine.

From Nature 27 September 1947. 
stratification; examples include the atmospheric tropopause and the oceanic thermocline. In the Earth's core, convection tends to homogenize the fluid so the results of stratification are minor; instead, the effects stemming from rotation create a barrier. Rotation imparts a dynamical rigidity to a homogeneous fluid in the direction parallel to the rotation axis, through a principle known as the Taylor-Proudman effect. Rotation causes the fluid to circulate in coherent vortices as illustrated in Fig 1. Because the vortices resist stretching or bending, fluid in the outer core is inhibited from crossing an imaginary cylinder tangent to the equator of the solid inner core, the so-called tangent cylinder.

The main difference between the models comes in setting the boundary conditions between the inner and outer cores, which has dramatic effects on the tangent-cylinder barrier and on azimuthal flow in the outer core (Fig. 1a compared with Fig. 1b). When Kuang and Bloxham take the same rigid-wall boundary conditions on the inner core as used by Glatzmaier and Roberts, they obtain a similar dynamo - that is, one which features an intense polar vortex in each hemisphere inside the tangent cylinder and intense toroidal magnetic fields in that region. Through coupling with these polar vortices, the inner core is spun into super (prograde) rotation relative to the mantle and crust, as first predicted in the Glatzmaier-Roberts model. When Kuang and Bloxham change the boundary conditions to free-slip, in an attempt to reduce the effects of viscosity in the model and make the balance of forces more like the inferred conditions in the Earth, the polar vortices are largely suppressed and the toroidal field is induced by the azimuthal flow outside the tangent cylinder that gives rise to a westward geomagnetic drift. In this case, anomalous rotation of the inner core is weaker and features episodes of both retrograde and prograde relative motion.

Is there geophysical evidence for a barrier $(6,000 \mathrm{~km}$ tall, according to theory) at the inner-core tangent cylinder? Surprisingly, there is. Most of the magnetic field that forms the Earth's exterior dipole field emerges from the core in flux patches located where the tangent cylinder intersects the core-mantle boundary ${ }^{4}$, just the location predicted by convective dynamo models. Even more dramatic evidence comes from the seismic detection of the super-rotation of the inner core ${ }^{5,6}$, which preliminary investigations indicate rotates faster than the mantle and crust by $1-3^{\circ}$ per year. This is close to the rate predicted by the dynamo models using rigid boundary conditions, a strong point in their favour. As more seismological data become available, however, the estimates of the anomalous innercore rotation will be subject to revision, so the final verdict is not yet in.

Realistic experiments on the mechanisms of planetary dynamos will remain out of reach for now, so numerical simulations provide the only way forward. Perhaps the most heartening outcome of the comparison of these two models ${ }^{2,3}$ is that it shows that such simulations are becoming increasingly powerful and accurate tools for relating magnetic fields to the dynamics in planetary interiors.
Peter Olson is in the Department of Earth and Planetary Sciences, Johns Hopkins University, Baltimore, Maryland 21218, USA.

e-mail:olson@gibbs.eps.jhu.edu

1. Kivelson, M. G. et al. Nature 384, 537-541 (1996).

2. Kuang, W. \& Bloxham, J. Nature 389, 371-374 (1997).

3. Glatzmaier, G. A. \& Roberts, P. H. Science 274, 1887-1890 (1996)

4. Bloxham, J. \& Jackson, A. J. Geophys. Res. 97, 19537-19563 (1992).

5. Song, X. D. \& Richards, P. G. Nature 382, 221-224 (1996).

6. Su, W.-J., Dziewonski, A. M. \& Jeanloz, R. Science 274, 1883-1885 (1996).

\section{The structure of starch}

\section{Paul Calvert}

S tarch granules provide an insoluble but readily biodegradable storage system for plants. This balance of mechanical stability with degradability arises somehow from the chemical structure of the amylose and amylopectin that make the substance up, and from the arrangement of amorphous and crystalline zones within the granules. Starch also has considerable potential as a biodegradable plastic, but we need to understand the structure better before we can approach the ideal of a controllable service life followed by rapid degradation. A new high-resolution X-ray study by Waigh et $a l{ }^{1}$ allows us for the first time to track across the structure of an individual granule.

Structural polysaccharides, such as starch, cellulose and chitin, undergo simultaneous polymerization and crystallization. This poses a structural constraint: how does the enzymatic machinery convert dissolved monomers into a solid polymer without getting choked by the solid as it forms? In chitin, little is known about how the solid is laid down. Cellulose, in contrast, has been much studied, and is extruded by an organelle that traces a spiral across the cell surface. Starch has the added constraint that it must lend itself to later controlled depolymerization, as it is used to fuel plant growth. It might be formed by a retreating enzymatic peripheral layer, or by entrained enzymes.

Because starch is such an important product, one would assume that the inner structure of granules had long since been analysed to death by electron microscopy. This has been done ${ }^{2}$ but there are problems with artefacts, which have always dogged electron microscopy of polymers. The radiation intensity in a transmission electron microscope is so high that organic structures are usually destroyed in seconds. Hence there is always a concern about artefacts due to beam damage and, for starch, due to loss of water during preparation.

In the new study, pure and intense radiation from the ESRF Synchrotron in Grenoble was used in a $2-\mu \mathrm{m}$-wide $\mathrm{X}$-ray beam that could be scanned across single granules to show the structure. It turns out that starch granules grow in rings, alternating between amorphous amylose and crystalline amylopectin (Fig. 1). The amylopectin crystals are built of double helices, which are arranged radially in the granule, so that the crystals are tangential to the surface.

In contrast, the spherulite structure found in most synthetic crystalline polymers has radial crystals, with the polymer chains running tangential to the surface. Such tangential chains make sense if they are thought of as adsorbing to the existing solid surface of a sphere. The radial chains seen in starch suggest, on the other hand, that monomer is being added to growing ends sticking out of the surface. So the structure of starch is determined by polymerization kinetics, not crystallization kinetics, which means that it will be difficult to arrive at an equivalent structure in a synthetic moulded part.

In natural polymers, the amorphous

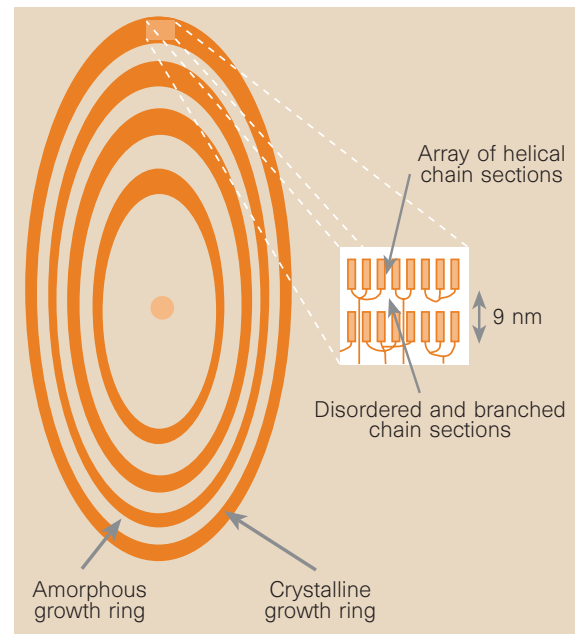

Figure 1 A schematic starch granule, as mapped by X-rays. Waigh et al. ${ }^{1}$ see alternating shells of amorphous and crystalline material, with the amylopectin helices that form the crystal lying perpendicular to the shell. They also find that the granules are ellipsoidal rather than spherical, so the helices do not point to a single focus, implying that some elongated structure must initiate the growth. 